\title{
Using fibre property measurements to predict the tensile index of microfibrillated cellulose nanopaper
}

\author{
Lewis Taylor (D) Jonathan Phipps • Stuart Blackburn • Richard Greenwood • \\ David Skuse
}

Received: 1 December 2019/Accepted: 9 May 2020/Published online: 25 May 2020

(C) The Author(s) 2020

\begin{abstract}
A wide variety of wood and non-wood cellulosic fibre sources were used as a feed to produce microfibrillated cellulose (MFC) using a grinding process. Nanopaper was formed using this product, and the tensile index was measured. The hemicellulose content of the feed fibres was measured, and was found to correlate with the production of finer microfibrils and a higher MFC tensile strength. The correlation with tensile strength was improved by the inclusion of a measurement of the MFC particle lengths as measured by a fibre image analyser, with the resulting relation fitting a modified Page Equation. It was hypothesised that the frequency of flaws in the feed fibre cross-section influences the length of the MFC particles produced, and so the zero-span tensile index of the fibres was measured as a proxy for this since it forces cross-sectional fibre breakage. The fibre zerospan tensile index was found to correlate with MFC particle length and so was used in its place in the
\end{abstract}

Electronic supplementary material The online version of this article (https://doi.org/10.1007/s10570-020-03226-2) contains supplementary material, which is available to authorized users.

L. Taylor · S. Blackburn · R. Greenwood · D. Skuse School of Chemical Engineering, University of Birmingham, Birmingham B15 2TT, UK

L. Taylor $(\bowtie) \cdot$ J. Phipps $\cdot$ D. Skuse

Par Moor Centre, FiberLean Technologies, Par Moor

Road, Par PL24 2SQ, UK

e-mail: 1xt189@bham.ac.uk; lewis.taylor@fiberlean.com equation. The resultant equation can predict MFC tensile strength from zero-span tensile index and hemicellulose content measurements of cellulosic fibres and can aid in optimising feedstock selection for mechanical MFC production processes.

Keywords Microfibrillated cellulose · Hemicellulose $\cdot$ Zero span tensile strength $\cdot$ Tensile strength

\section{Introduction}

Microfibrillated cellulose

Cellulose is an essential structural component in higher plants and is the most abundant polymer on Earth, with an almost inexhaustible supply of around $1.5 \times 10^{12}$ tonnes per year produced by biomass (Klemm et al. 2005). Cellulose fibres can be separated from other biomass components by pulping and bleaching processes, producing a cellulose fibre pulp that is used primarily in the production of paper and cellulose-based packaging to impart mechanical strength properties.

These mechanical properties are a consequence of the fibre microstructure. Cellulose fibres have a fractal-like structure with several hierarchies of fibre-like sub-units that extend down to the nanoscopic scale. A cellulose fibre consists of parallel chains of 
long fibrils with micron-scale diameters, which are similarly made up of microfibrils around $30 \mathrm{~nm}$ in diameter. These microfibrils in turn consist of elementary fibrils around $3.5 \mathrm{~nm}$ in diameter (ChingaCarrasco 2011).

In 1982, Turbak et al. (1983) and Herrick et al. (1983) reported that cellulose fibres could be disintegrated to liberate these finer structural components, greatly increasing the specific surface area and hydrogen bonding capability. This product was termed microfibrillated cellulose (MFC), and has been found to have many applications that exploit its hydrophilicity, renewability, biodegradability, and high specific strength and surface area (for reviews regarding applications, see Klemm et al. 2011; Shatkin et al. 2014).

The original production process involved disintegrating cellulose pulp fibres into MFC using a homogenizer (Turbak et al. 1983). In the decades since, other equipment has been used as an alternative, such as microfluidizers and friction grinders, and chemical pre-treatment methods including enzymatic degradation and 2,2,6,6-tetramethylpiperidine-1-oxl radical (TEMPO) oxidation have been applied to reduce the energy input (for a review on production methods, see Siró and Plackett 2010).

FiberLean Technologies has adapted minerals grinding technology to mechanically disintegrate cellulose fibres into MFC cost-effectively at large scales, without requiring cellulose pre-treatment. This method uses stirred media detritor technology (Husband et al. 2015), which disintegrates fibres into MFC by agitating grinding media beads. In this process, a paper filler mineral such as calcium carbonate is added as a grinding aid, greatly reducing the energy required. A laboratory scale equivalent of this process is used to produce the MFC that is the subject of this research.

MFC has been made from numerous sources including hardwoods (e.g. birch, eucalyptus, and acacia), softwoods (various species of coniferous trees) and non-wood sources (e.g. cotton, abaca, flax, bamboo, and sugar beet (Jonoobi et al. 2015). The fibre source can have a strong influence on MFC quality. For example Alila et al. (2013) found that abaca and sisal fibres produced finer MFC than flax and hemp. Others have investigated the influence of fibre species on MFC quality (e.g. Chaker et al. 2013; Desmaisons et al. 2017) but these are limited to comparisons of a small number of species at a time, making the drawing of conclusions applicable to most fibre sources problematic.

Fibres can differ from each other in many ways, so it cannot be taken for granted that differences in MFC quality between two fibre sources are due to a difference in one parameter rather than another. This work therefore analyses twenty-four different fibre species in order to have a large enough data set that such comparisons are more valid, and so the relative importance of a fibre property can be assessed with a greater degree of confidence.

Paper tensile strength theory

The MFC produced by the FiberLean process is in the form of a MFC-mineral composite that was originally created to service the paper filler additive market. In this application, the addition of MFC to a paper formulation increases the specific strength, allowing for a disproportionate amount of native fibres to be removed and replaced with paper filler mineral; this reduces the cost of the paper considerably and improves optical properties. The MFC quality is assessed by forming nanopaper sheets and testing their tensile strength; this strength appears proportional to the specific strength increase that this MFC would impart on paper when added to a fibre furnish.

Page (1969) introduced what is today the most commonly used theory for predicting the tensile strength of paper. This postulates that the failure of a sheet occurs partly by bonds between fibres being broken, and partly by the fibres themselves breaking across their cross-sections, and that the tensile index of the sheet is more dependent on the weakest of these two mechanisms. The Page Equation describing this is stated below:

$\frac{1}{T}=\frac{9}{8 Z}+\frac{12 A \rho}{\tau_{B} P L(R B A)}$

where $T$ is the sheet tensile index $(\mathrm{Nm} / \mathrm{g}), Z$ is the zero-span tensile index $(\mathrm{Nm} / \mathrm{g}), A$ is the fibre crosssectional area $\left(\mathrm{m}^{2}\right), P$ is the fibre cross-section perimeter $(\mathrm{m}), \rho$ is the fibre density $\left(\mathrm{kg} / \mathrm{m}^{3}\right), \mathrm{L}$ is the fibre length $(\mathrm{m}), \tau_{B}$ is the shear bond strength per unit area $(\mathrm{Pa})$, and RBA is the relative bonded area.

Zero-span tensile index is a measure of individual fibre strength and is discussed later in this work. RBA is a measure of the fraction of the fibre surface area that is used for inter-fibre bonding. The first term on 
the right-hand side represents the weakness of the individual fibres, whereas the second term represents the weakness of the bonds between fibres. Usually, a sheet of paper fails due to bonds between fibres breaking rather than the fibres themselves breaking, so the second term is limiting. Adding MFC to a fibre furnish greatly increases relative bonded area (Lindström et al. 2016), and so tensile index tends to improve considerably.

\section{Hemicellulose}

Fibre chemical composition is known to be important in papermaking. Most raw plant material from which cellulose fibres are extracted are rich in hemicellulose. Though pulping and bleaching removes much of the hemicellulose, there is still typically a large residual fraction within the fibre cell wall, with the amount dependent on fibre species and pulping conditions.

Hemicellulose is a broad term for a wide variety of polysaccharides with differing monomer sugars, functional groups, and degrees of branching, but for woods and many non-woods, there are only two important families; xylans and glucomannans. Xylans are found in the vast majority of plants, and account for almost all the hemicellulose in hardwoods, whereas glucomannans are found in large quantities in softwoods (in comparable amounts to xylans) (Ebringerová 2005). Compared to cellulose, hemicellulose is always amorphous, whereas cellulose is partly crystalline, and hemicellulose molecules have relatively short chain lengths of 70-200 units (Fengel and Wegener 1983), compared to 300-1700 units typical for cellulose (Klemm et al. 2005).

Within a fibre cell wall, hemicellulose closely associates with the cellulose microfibril surface, forming a layer separating neighbouring microfibrils. NMR spectroscopy work by Liitiä et al. (2003) indicates that both xylan and glucomannan do this, and are comparable in function. Hemicellulose has a branched, amorphous structure, and readily swells in water, as shown by work investigating the change in zeta potential during this process (Uetani and Yano 2012). This hydrophilicity also aids in the plasticity of the fibre to deformation (Bolam 1965), which would be expected to facilitate disintegration into MFC.

NMR studies by several authors using fibres that have undergone different pulping conditions have shown that reducing the hemicellulose content appears to increase the fibril aggregate dimension size appreciably (Hult et al. 2001; Virtanen et al. 2008; Duchesne et al. 2001). This supports the notion that hemicellulose inhibits the spontaneous coalescence of neighbouring microfibrils.

Hemicellulose has long been understood to aid in conventional fibre refining for papermaking; chemically removing the hemicellulose content prior to refining reduces the tensile strength of the refined fibres (Bolam 1965). Sorbing hemicellulose onto fibres prior to refining has been found to improve sheet tensile strength, primarily by reducing the 'kink' deformations induced in the fibres (Mäkelä et al. 2010).

It is therefore plausible that a higher hemicellulose content would lead to high quality MFC. Existing work in the literature has supported this idea. Iwamoto et al. (2008) showed that drying a pulp after removing hemicellulose by alkali treating results in irreversible microfibril aggregation, inhibiting fibrillation compared to an untreated pulp. Numerous authors have found that a high hemicellulose content coincides with a high microfibril yield and better individualisation. This appears true whether comparing fibres from different plant species (Alila et al. 2013; Desmaisons et al. 2017; Chaker et al. 2013) or from the same plant species but with different pulping conditions (Chaker et al. 2013; Petroudy et al. 2015; Spence et al. 2010).

There are two major mechanisms thought to explain this. The presence of surface hemicellulose itself improves inter-fibre bonding (or inter-fibril bonding in the case of MFC), since amorphous hemicellulose chains extend out from the microfibrils when immersed in water, and form bridges between neighbouring microfibrils when dried (Bolam 1965). Therefore, when disintegrating a high hemicellulose pulp into MFC, the liberated surface area has a higher surface concentration of hemicellulose, and so this strengthening effect is enhanced compared to a low hemicellulose pulp. Arola et al. (2013) removed hemicellulose from nanocellulose with xylanase enzymes, which resulted in poorer tensile properties, even with similar nanocellulose geometry, clearly demonstrating this effect.

The second mechanism is that a high hemicellulose pulp produces finer microfibrils with better individualisation, as microscopy images in various studies has demonstrated (e.g. Alila et al. 2013; Iwamoto et al. 2008; Chaker et al. 2013). Given similar microfibril 
lengths, this increases particle aspect ratio, improving tensile strength. Hemicellulose forms an amorphous layer between microfibrils that readily swells in water, and so this would be expected to provide a preferred plane of breakage parallel to the microfibril lengths, thereby facilitating the production of finer microfibrils. Additionally, xylan develops a surface charge due to carboxyl group dissociation under typical processing conditions, causing mutual microfibril repulsion, enhancing this effect to some degree.

Due to the importance of hemicellulose indicated so far in the literature, and its expected influence on MFC geometry and bonding, the hemicellulose content was investigated for all fibre species tested in this study.

\section{Zero-span tensile strength}

It is later suggested that the fibril lengths of the MFC correlate with a high MFC tensile strength. It is desirable to be able to predict the resultant MFC fibril length from intrinsic fibre properties. It is reasonable to believe that having long fibrils within the fibre structure will, all other things being equal, lead to long liberated fibrils. Also, having fibrils with few preexisting defects should reduce the degree of fibril length degradation during processing. Both of these factors are expected to affect the individual fibre strength; intrinsically long fibrils result in fewer discontinuities at fibril endpoints, and having undamaged fibrils means fewer microscopic weak points in the fibre. Both can be seen to influence the 'quality' of the fibre cross-sectional area, i.e. having long, undamaged fibrils should result in the cross-sectional area having few flaws.

A measurement that could assess the specific strength of the fibre cross-sectional area could therefore be useful for indicating the frequency of fibril flaws and intrinsic fibril length; and is therefore expected to correlate with long fibril lengths of the MFC product. The zero-span tensile index of a fibre sheet prior to MFC production is believed to be such a measurement. In the zero-span tensile test, the two clamps are essentially touching (within microns of each other), forcing the vast majority of the fibres between the clamps to be held by both clamps at once, since the separation distance between clamps is a small fraction of typical fibre lengths. When the sample is broken under tensile stress, these clamped fibres will fail by cross-sectional breakage, rather than the bonds between fibres failing as with conventional tensile testing. Since the zero-span tensile test is normalised by weight, the thickness of the fibre cell wall and fibre diameter are accounted for.

The notion that zero-span tensile strength is a measure of fibre damage is well-supported. Zeng et al. (2012) damaged fibres by refining, finding that the zero-span tensile strength was inversely proportional to the frequency of fibre kinks induced (i.e. sharp bends in the fibre). Joutsimo et al. (2005) induced cell wall damage (dislocations) by homogenization, which decreased fibre strength; they attributed this to inducing non-uniform load distributions across the crosssection.

Research has shown that fibril and microfibril-scale damage is also important. Nevell and Nugawela (1987) treated fibres with acids, causing localised damage to microfibrils that substantially reduced zerospan tensile strength. Wathén (2006) damaged fibrils homogeneously throughout the fibre by thermal ageing degradation, and found that the zero-span strength decreased.

\section{Definition of research objectives}

MFC is produced by a mechanical process with a large and diverse range of fibre species, and attempts are made to correlate the tensile index of this MFC with the feed fibre properties, including the fibre hemicellulose content, and the fibre zero-span tensile index.

\section{Materials and methods}

\section{Fibre sources}

In total, twenty-four separate fibre species were investigated, from a variety of wood and non-wood sources. These are listed in Table 1 below.

Pulp preparation

Fibres were added to the grinding process as a $30 \%$ solids cake. Miscanthus, sorghum, bagasse, enzymetreated Nordic pine, and Giant Reed were supplied in this form, whereas most other fibre sources were supplied as dry pulp boards. These dry pulps were soaked in water and broken down into individual fibres using a pulp disintegrator for $10 \mathrm{~min}$. Excess water 
Table 1 Fibre species used in this study

\begin{tabular}{lll}
\hline Softwoods & Hardwoods & Non-woods \\
\hline Nordic pine & Birch \#1 & Cotton (linters) \\
Black spruce & Birch \#2 & Jeans: \\
Radiata pine & Eucalyptus & Abaca \\
Southern pine & Acacia & Sisal \\
Douglas Fir & Mixed South Asian Hardwood & Kenaf \\
Enzyme-treated Nordic Pine & Mixed European Hardwood & Giant Reed (Arundo Donax) \\
Dissolving Pulp* & Tissue Dust $\dagger$ & Bagasse (sugar cane) \\
& & Miscanthus \\
& & Sorghum
\end{tabular}

* Dissolving Pulp is a Nordic pine/spruce blend (Domsjö Fabriker AB-Domsjö Cellulose Plus) that was subjected to the sulphite bleaching process, rather than the kraft (sulphate) process which all other softwoods and hardwoods listed underwent

$\dagger$ Waste short fibres collected from the dust extraction system of a tissue mill

†Cotton fibres from recycled jeans, following disintegration into individual fibres and formation into a pulp board

was removed by a vacuum filter, forming a cake. To avoid choking of the grinder due to entanglement of very long fibres, the flax fibres were cut with a guillotine into fragments around $3 \mathrm{~mm}$ in length.

MFC production by stirred media detritor grinding

MFC was produced from these fibres using a lab-scale version of the FiberLean production process. This involves a grinder that uses the motion of media beads to disintegrate the fibres. The presence of micron-scale mineral particles greatly improves the efficiency of this process, so the fibre charge was complemented with an equal amount (on a dry mass basis) of IC60 (60 $\mathrm{wt} \%<2 \mu \mathrm{m})$ ground calcium carbonate mineral (Imerys). Sufficient water was also added to form a slurry with the target fibre solids content. The precise operating conditions, including the energy input, are proprietary. When a grind was completed, the slurry was separated from the media with a vibrating screen, or by a washing method when particles were too coarse for the screen.

Previous experience with this process has shown that almost all fibre sources reach a peak tensile strength at around the same energy, and an unpublished investigation with seven of the fibre species in listed in Table 1 has confirmed this. The relationships between energy input and MFC tensile strength for these seven fibre species are displayed in the Energy
Sweeps tab in the Supplementary Material. Consequently, a single energy input was chosen which was applied to all fibre species listed in Table 1 to produce MFC.

Nanopaper sheet preparation

The MFC-mineral composite product was collected as an aqueous slurry. The solids content was measured by weighing a sample before and after oven drying. The mineral content was determined from the change in weight of an oven-dried sample after burning off the cellulose in a $450{ }^{\circ} \mathrm{C}$ furnace for $2 \mathrm{~h}$. Using this information, sufficient IC60 calcium carbonate mineral was added to the sample to dilute the MFC content to $20 \mathrm{wt} \%$ on a dry mass basis. These samples were used to form a nanopaper sheet in a vacuum sheet former and dried using a Rapid-Köthen dryer, targeting a weight of $220 \mathrm{gsm}$. These sheets were conditioned in a controlled environment at $23{ }^{\circ} \mathrm{C}$ and $50 \%$ relative humidity overnight prior to tensile testing.

Nanopaper tensile testing

The nanopaper samples were weighed and cut into strips $15 \mathrm{~mm}$ wide. These strips were clamped in a Testometric M350 tensile tester and strained at a constant rate until failure. The force applied at break was divided by the strip width and gsm to obtain the 
tensile index in $\mathrm{Nm} / \mathrm{g}$. The tensile index of this MFCmineral composite nanopaper is much weaker than pure MFC due to the mineral disrupting inter-fibril bonding. However, work with MFC made from this process by Phipps et al. (2017) has shown that the tensile indices of FiberLean MFC with or without a given fraction of mineral added are roughly proportional (i.e. breakage is mainly bonding-limited in both cases), so the conclusions are still believed to be applicable to pure MFC. Three nanopaper sheets were produced for each sample, each cut into five strips. The mean tensile index of these fifteen strips is reported as the sample tensile index here. Fibre content was verified to be on target by measuring the ash content after burning off the cellulose in a $450{ }^{\circ} \mathrm{C}$ furnace for 2 $\mathrm{h}$ (these results are displayed in the Tensile Sheet Mineral Content tab in the Supplementary Material).

Fibre image analysis

A Valmet FS5 fibre image analyser was used to measure geometric parameters of the original fibres before grinding, and the MFC particles after grinding. This equipment operates by pumping a sample suspension past a camera that takes thousands of images, and parameters such as length and width distributions are determined by a computer algorithm. Though designed for fibre measurement, this is sensitive enough to measure MFC particles with lengths around a hundred microns and above.

\section{Hemicellulose content}

Hemicellulose measurements were subcontracted to Labtium Oy, who used the SCAN-CM 71:09 method (Scandinavian Pulp, Paper and Board Testing Committee 2009). Fibre samples underwent sulphuric acid hydrolysis, and the resultant concentration of various monomer sugar residues were detected by chromatography. This included glucose, xylose, mannose, arabinose, and galactose. To convert these sugar contents into xylan and glucomannan hemicellulose contents, it was assumed that the xylan content was the sum of all the xylose and arabinose, and the glucomannan content consisted of all the galactose, mannose, and 1 unit of glucose for every 4 units of mannose, since a ratio of 1:4 glucose to mannose is typical in softwood glucomannan (Ebringerová 2005). Total hemicellulose content was determined by adding together the xylan and glucomannan fractions. The hemicellulose content of cotton linters (which is negligible) was also used as the hemicellulose content of the jeans cotton, since it was expected that the presence of dye and other additives in the jeans cotton would give misleading results. This is a reasonable assumption as textile-grade cotton contains negligible hemicellulose (Prado and Spinacé 2015).

\section{Lignin content}

Acid-soluble and acid-insoluble lignin content of the pulps were measured by Labtium Oy according to a modified TAPPI standard $T 222 \mathrm{om}-88$ test.

Fibre zero-span tensile testing

Zero-span tensile tests were carried out on the initial fibre samples rather than the MFC. The TAPPI standard $T 231 \mathrm{~cm}-07$ (Technical Association of the Pulp and Paper Industry 2007) was followed, using $60 \mathrm{gsm}$ mineral-free handsheets for each fibre species produced according to TAPPI standard T $205 \mathrm{sp}-12$ (Technical Association of the Pulp and Paper Industry 2012). Each handsheet was cut into five strips, which were clamped in a Pulmac zero-span tensile tester and strained until broken. As with conventional tensile testing, the force measured was converted to a zero-span tensile index. Four dry sheets were tested for each fibre species, and a mean average result is reported here.

Scanning electron microscopy

A Jeol 6700 Scanning Electron Microscope was used to image several MFC samples at 10,000 x magnification. Preparation involved filtering a dilute MFC sample through a $0.2 \mu \mathrm{m}$ nucleopore membrane. The samples were sputter coated with platinum to form a conductive monolayer. Secondary electrons emitted from the sample during measurement were used to form the images. 


\section{Results and discussion}

Influence of hemicellulose content

Hemicellulose within bleached chemical pulp resides within the fibre cell wall, where it forms a layer around the microfibrils, separating them from their neighbours. It was expected both from mechanistic considerations and from results previously reported in the literature that it would positively correlate with MFC quality. The hemicellulose content for each fibre species was measured, and is plotted against the MFC tensile index as Fig. 1.

Figure 1 shows that a high hemicellulose content of the fibre correlates with a high MFC tensile index. Fitting the data to a general straight line equation gave an $\mathrm{R}^{2}$ value of around 0.63 , which is moderately good for a single parameter, considering that cellulose is a natural product with a complex hierarchy of structures with different scales and chemistries between plant species.

As discussed in the introduction, there are two mechanisms by which hemicellulose is thought to improve MFC tensile index. One mechanism is that a high hemicellulose fibre when disintegrated to form

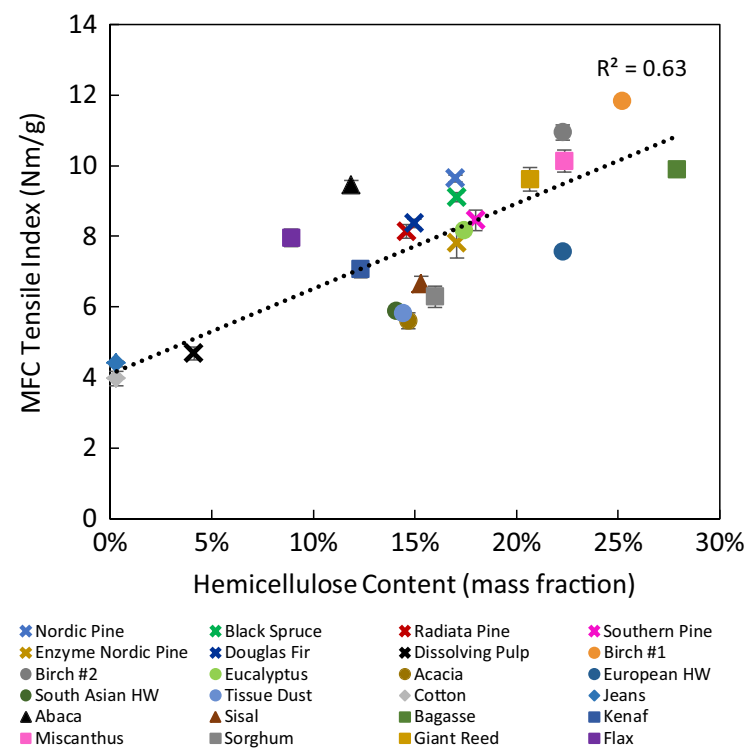

Fig. 1 Fibre hemicellulose content (wt $\%$ ) versus tensile index at the selected energy input. For this figure and all subsequent plots, crosses represent softwoods, circles represent hardwoods, diamonds represent cotton fibres, triangles represent leaf fibres, and squares represent other miscellaneous non-wood sources
MFC has more of this liberated surface area coated in hemicellulose, which is thought to form bridges between particles upon drying and thereby improve relative bonded area and specific bonding strength. The other mechanism is that the presence of hemicellulose, being a relatively mechanically weak amorphous layer, provides a preferred plane of breakage along the microfibril lengths, thereby facilitating disintegration into finer microfibrils, increasing the aspect ratio. To assess whether the latter mechanism is taking place, SEM images were taken of samples with a range of hemicellulose contents to assess fibril and microfibril diameters. Four of such images are displayed as Fig. 2.

Figure 2a shows bagasse MFC, which had the highest hemicellulose content of any fibre tested, at $28 \%$. There appears to be a much higher frequency of finer microfibrils in this case than dissolving pulp and cotton MFC, shown as 2c, d, which have $4 \%$ and $0 \%$ hemicellulose respectively. Nordic pine MFC with a hemicellulose content of $17 \%$ is displayed as $2 b$, and also shows a high abundance of fine microfibrils, though to a lesser extent than bagasse MFC.

A comparison of Fig. 2b, $c$ is important; the dissolving pulp used in the latter is a Scandinavian pine/spruce blend, similar to the Nordic pine used in the former; the major difference is in their processing. The Nordic pine pulp underwent a kraft pulping process, which cannot penetrate well into the fibres to remove hemicellulose, whereas the dissolving pulp was pulped with the sulphite process, which is capable of this, removing the vast majority of the hemicellulose. Consequently, the fact that the liberated microfibrils in Nordic pine MFC tend to be much finer than with dissolving pulp MFC implies that the correlation of hemicellulose content with finer microfibrils is causal.

There is also the question of whether the two dominant types of hemicellulose found in these fibres, xylan and glucomannan, are equivalent in terms of enhancing microfibrillation. Correlations between MFC tensile strength and xylan or glucomannan alone, or a weighted sum of the two were investigated, but the best fit was found by treating the two as identical and correlating tensile strength with the sum of the two species. These data are displayed in the Pulp Chemical Composition tab in the Supplementary Material. 

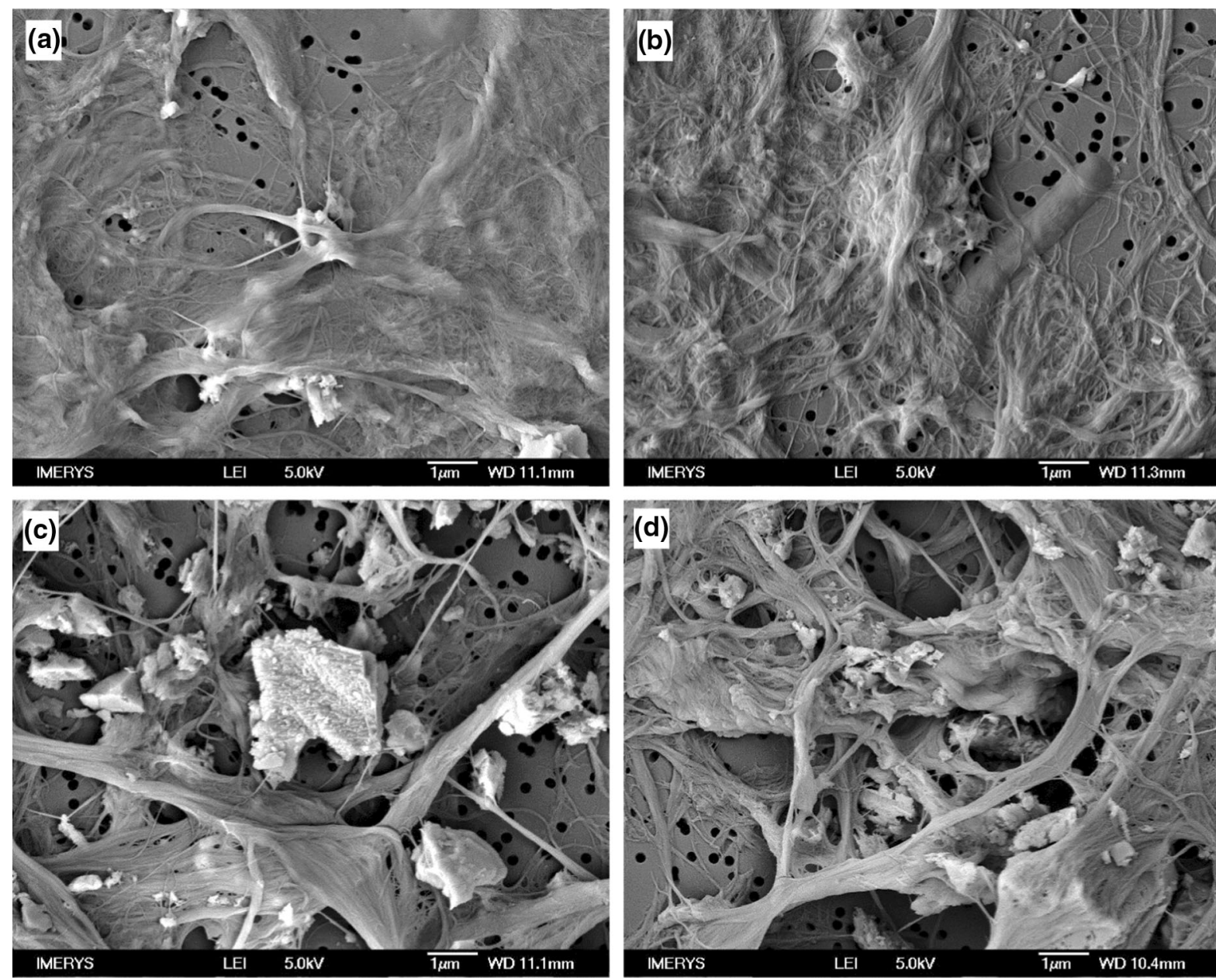

Fig. 2 Secondary electron SEM images of MFC-mineral composite made from a bagasse (28\% hemicellulose), b Nordic pine ( $17 \%$ hemicellulose), c dissolving pulp ( $4 \%$

Influence of lignin content

Given that the influence of hemicellulose is established, it was an open question as to what the influence of the lignin content would be. For thermomechanical or chemithermomechanical pulp, there is an extensive lignin coating on the fibre surface, which seriously inhibits the ability of the fibre to fibrillate and form high quality MFC, at least for entirely mechanical processes (e.g. Meyer et al. 2018; Lahtinen et al. 2014). However the role of lignin within the fibre cell wall is less clear, since good quality MFC can still be made at high lignin contents with unbleached chemical pulp (e.g. Spence et al. 2010).

hemicellulose $)$ and d cotton ( $0 \%$ hemicellulose). The low hemicellulose fibres give coarser and more strongly defined fibrils and microfibrils

Especially considering the numerous relatively novel non-wood pulps of unknown chemistry used in the present study, it was initially conceivable that differences in lignin content could be contributing to the noise seen in Fig. 1. The lignin contents of the fibre species used in this study were measured, and the results are shown in the Pulp Chemical Composition tab in the Supplementary Material. Lignin was found to be present only at very low concentrations in all samples, except Giant Reed, which contained 12.7\% lignin and does not seem to perform unusually compared to other fibre species. Therefore, for this study, the influence of lignin on these results can be neglected. 
Influence of feed fibre geometry

Fibre geometry has a strong influence in tensile strength in paper made from cellulose fibres; all other things being equal, longer and thinner fibres result in higher tensile strengths because they maximise aspect ratio. Longer fibres increase the number of connections that each individual fibre can make, and thereby distribute stress over a larger area. Finer fibres (with a thinner cell wall) result in more fibres per unit mass. The fact that a high fibre aspect ratio leads to high sheet tensile strength has been found experimentally for both softwoods (Horn 1974) and hardwoods (Horn 1978). The Page Equation, shown as Eq. (1) earlier in this paper, makes use of such fibre dimensions to model paper tensile strength.

Though established as true for fibres, the influence of feed fibre dimensions on MFC properties was an open question. It stands to reason that in the case of partially processed MFC where the initial fibre structure is partly intact that this relationship would hold somewhat, but it is intuitive that as the fibre structure degrades into fully processed MFC, such a correlation would disappear. It was not ruled out, however, that the dimensions of the fibre such as length or cell wall thickness affect how the fibre disintegrates into MFC.

Geometric parameters of the fibres were measured using a fibre image analyser, including length and length distributions, widths, and coarseness (a measure of fibre wall cross sectional area). Attempts were made to plot such geometric parameters against tensile index, and no clear correlation was apparent. The geometry of the feed fibres is therefore not thought to have any obvious influence on the quality of fully processed MFC. The geometric parameters measured by the fibre analyser and their near-zero correlations with MFC tensile strength are displayed in the Pulp Geometry tab in the Supplementary Material.

\section{Influence of MFC length}

Stirred media detritor grinding produces MFC mostly in the form of fibril 'aggregate' particles rather than fully individualised fibrils, i.e. the fibrils are liberated from the fibre, but are imperfectly separated from their neighbours and so are physically rooted to other fibrils to form a network (The Microscopy tab in the Supplementary Material gives several examples of what this looks like for different fibre species). These particles are large enough to have some geometric properties inferred from measurement with the fibre analyser. In this study, the length-weighted fibre length of these MFC particles, called from here onwards MFC length was found to be useful. The fibre analyser interprets the 'fibre length' in this case as the longest dimension of an MFC particle. When plotting MFC length against MFC tensile index for all fibre species, there is no general correlation, but for fibres of a similar hemicellulose content, there appears to be a clear positive trend (see Figure D1 in the MFC Correlation Investigation tab in the Supplementary Material). Therefore, using the product of hemicellulose and MFC length to predict tensile index appeared promising.

In order to have some theoretical grounding for this correlation, the Page Equation was used as a starting point. The Page Equation, shown as Eq. (1) in the introduction, was formulated to predict tensile strength for straight, individualised fibres with lengths on the scale of millimetres, when formed into a mineral-free sheet. It is not obvious whether this would be directly applicable to MFC nanopaper, which is in the form of highly entangled and conformable, physically connected networks of fibrils, which in this study forms a composite that is $80 \%$ paper filler mineral. However, the Page Equation is conceptually useful since parameters such as fibre length and relative bonded area are expected to be applicable in a similar way to this MFC-mineral composite nanopaper. This equation will be used to justify a semi-empirical model to predict MFC tensile strength using hemicellulose and MFC length.

It stands to reason that as relative bonded area increases greatly with MFC production, the bonding term of the Page Equation becomes less limiting, and strength would eventually be dominated by the zerospan term representing fibre or fibril breakage if fibrillation is extensive enough. For chemical-treated nanocellulose (i.e. TEMPO MFC) with very fine microfibrils and very extensive bonding, this may be the case, but at least for the relatively coarse mechanically processed FiberLean MFC under consideration, further work not published here shows that this limit is not reached. It was found that the tensile strength of MFC produced using this method varies strongly with mineral content, demonstrating that bonding failure is still important. 
Additionally, the zero-span tensile strength of the MFC at 50 dry wt $\%$ mineral was measured, and for most fibre species was over 2.5 times the long span tensile strength of the MFC, when normalised by fibre content. This MFC zero-span tensile strength was also found to vary strongly with mineral content, implying that bonding failure was not completely suppressed (likely because smaller MFC particles are more prone of slipping out of the gap between the clamps). The notion that MFC fails mainly by network failure even in a zero-span tensile test is supported by electron microscopy of breakage sites by Varanasi et al. (2012). This makes the measured MFC zero-span strength a gross underestimate of the true value, and so fibril failure is responsible for much less than $40 \%$ of the breakage.

Therefore, the fibre weakness term will be assumed to be zero as the MFC zero-span tensile strength is believed to be much higher than the bonding strength. The Page Equation is therefore reduced to:

$\frac{1}{T}=\frac{12 A \rho}{\tau_{B} P L(R B A)}$

Hemicellulose on microfibril surfaces would be expected to increase relative bonded area since extended hemicellulose chains allows for more extensive and intimate contact between microfibrils. Additionally, hemicellulose appears to result in finer microfibrils when MFC is produced. Finer diameter microfibrils could be expected to be more flexible, and so be more capable of deforming for a more intimate contact with other particles. It is also expected that finer microfibrils would be more susceptible to capillary forces drawing them in contact with other surfaces during drying. These effects would imply that a high hemicellulose content would lead to a high relative bonded area. It is conceivable that these same factors could lead to a higher specific bond strength $\tau_{B}$ within a given region of bonded area (i.e. more hemicellulose chains likely means a higher fraction of the bonded area in molecular contact).

The perimeter to cross-sectional area ratio, $P / A$, would also correlate with increased bonding; since this increases as fibril diameter decreases, this would also correlate with hemicellulose content. Though $P / A$, $R B A$, and $\tau_{B}$ are all believed to be influenced by hemicellulose content, their relative influences cannot be distinguished. If hemicellulose correlated linearly with all three, then it would be expected that, all other things being equal, tensile index would be proportional to the cube of the hemicellulose content; however, Fig. 1 shows that this correlation is linear, and the maximum $\mathrm{R}^{2}$ value of the fit between hemicellulose and MFC tensile strength was obtained with a hemicellulose exponent value close to unity. Therefore, rather than distinguishing between the three, the $R B A$ term, the $P / A$ term, and the $\tau_{B}$ term in the denominator of the Page Equation are replaced with the hemicellulose content $H$ in Eq. (3) below. Since the larger MFC particles are the primary loadbearing particles, the corresponding MFC length term $L_{M F C}$ is expected to be analogous to the fibre length term $L$ in the Page Equation, and so this substitution is also made:

$\frac{1}{T}=k \frac{12 \rho}{L_{M F C} H}$

where $k$ is a proportionality constant. Density $\rho$ is constant for all MFC, so can be combined with $k$ under a single coefficient $B_{1}$ :

$\frac{1}{T}=\frac{1}{B_{1} L_{M F C} H}$

which can be inverted to obtain the MFC tensile index:

$T=B_{1} L_{M F C} H$

The product of $L_{M F C}$ and $H$ was calculated for all fibre sources and plotted against MFC tensile index in Fig. 3. As can be seen, the fit to a general straight line is much better when MFC length is included in this analysis compared to just hemicellulose content alone, with the $\mathrm{R}^{2}$ improving from 0.63 to 0.87 . However, this fit requires an intercept that is not accounted for in Eq. (5). This intercept is at least in part because fibrillation can take place in the absence of hemicellulose, as the cotton linter and jeans MFC demonstrate. Other contributions to this intercept may be due to some of the assumptions being inaccurate, for example if the contribution of fibre weakness to the strength was significant, or if the tensile strength at $80 \%$ mineral loading was not directly proportional to mineral-free tensile strength for all samples. Despite this, the fit is good, and so an empirical residual strength term, $\sigma_{0}$, is added to Eq. (5), representing at least in part the MFC tensile strength when hemicellulose is absent. 


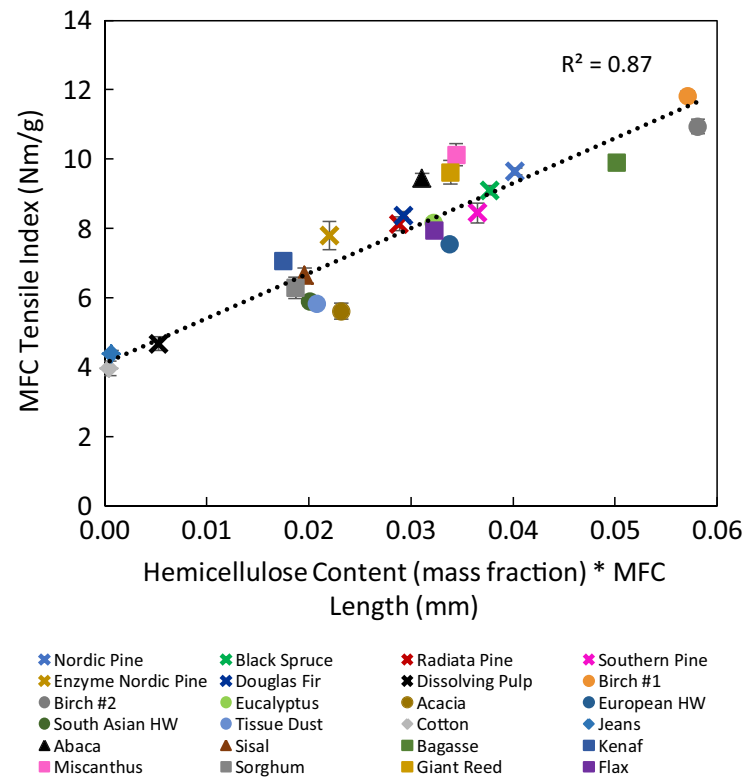

Fig. 3 Tensile index versus the product of the hemicellulose content and the MFC length

$T=B_{1} L_{M F C} H+\sigma_{0}$

Figure 3 gives $\sigma_{0}$ a value of $4.1 \mathrm{~N} \mathrm{~m} / \mathrm{g}$ on a basis of the fit with the twenty-four fibre sources tested.

Despite the good fit, using the MFC length in order to shortlist which fibre sources are worth using as a feed source is impractical; the MFC must first be created to obtain this length value, and so this only reduces the need of the tensile test, saving little effort overall. Instead, it is more useful to identify the reasons behind why different fibres give MFC of different MFC particle lengths, and relate this to a parameter that can be measured in the unprocessed fibres.

\section{Influence of fibre zero-span tensile index}

It stands to reason that if the fibrils that form the cell wall are long, that once the fibre is disintegrated the liberated fibrils will also be long. Additionally, a greater number of flaws in the fibril structure would make breaking the fibril (and the larger scale fibre) across the cross-section easier. Therefore, a fibre that has intrinsically long fibrils with few discontinuities and cross-sectional flaws would be expected to produce long fibrils when ground down into MFC, and would therefore give a relatively high MFC particle lengths as measured by the fibre analyser. As discussed in the introduction, the zero-span tensile test gives an indication of the frequency of these flaws by forcing fibres to break across their cross section, with a high value indicating a lack of flaws and discontinuities.

The zero-span tensile test is not solely a measure of fibre and fibril damage. A good correlation has been found between the microfibril angle and the zero-span tensile strength, at least with fibres that are relatively undamaged by processing (Courchene et al. 2006). This agrees with data and theoretical modelling from El-Hosseiny and Page (1975). However, the microfibril angle is not expected to directly influence intrinsic fibril lengths or frequency of fibril damage, and it would therefore be surprising if it influences fibril length in MFC. Although easy to measure in wood, the microfibril angle is difficult to measure accurately in pulped fibres, and so was not attempted in this study, particularly because it is impractical in an industrial setting. This is likely a significant cause of some of the data spread seen in Fig. 4.

Figure 4 shows the correlation between the fibre zero-span tensile index and the fibre length of the resultant MFC. There appears to be a linear relation between these two parameters when considering most fibre species, although there are several exceptions

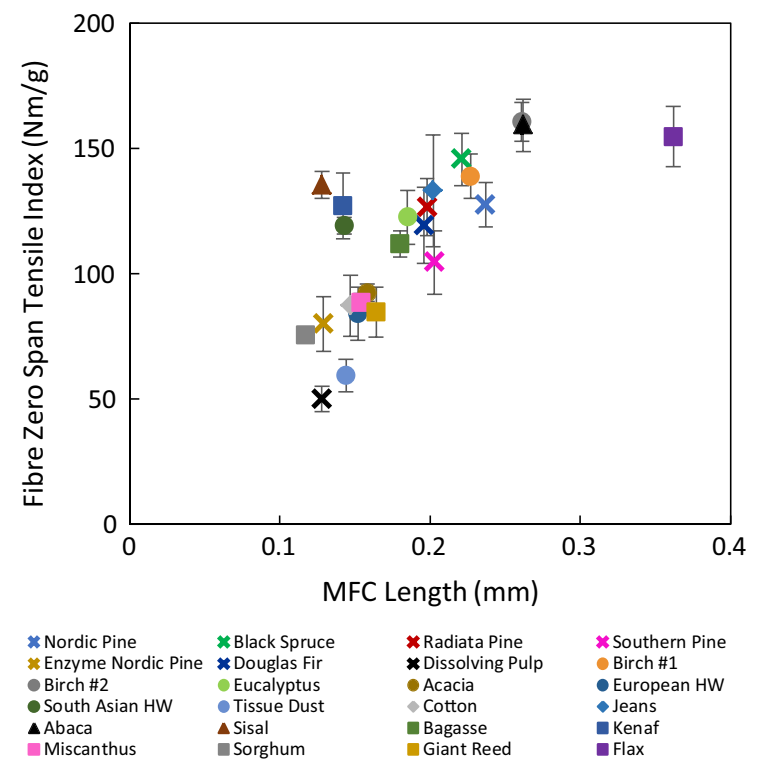

Fig. 4 The relationship between the zero-span tensile index of unground fibres and the resultant MFC length 
that deviate significantly, including flax, sisal, and kenaf.

Despite this complication, the fit between MFC length and zero-span tensile index for most fibre species appears sufficient for most of the other fibre species to use zero-span tensile index as a replacement for MFC length in Eq. (6). This equation is therefore modified below:

$T=B_{2} Z H+\sigma_{0}$

where $Z$ represents the zero-span tensile index of the fibre and $B_{2}$ is a proportionality coefficient. Figure 5 shows the correlation between the product of the fibre zero-span tensile index and hemicellulose content, with the MFC tensile index.

As Fig. 4 shows, the assumption that fibre zero-span tensile index correlates with MFC length is not completely accurate, so it is not surprising that the fit shown in Fig. 5 for Eq. (7) $\left(\mathrm{R}^{2}=0.78\right)$ is poorer than when MFC length was used $\left(R^{2}=0.87\right)$. Although this relationship fits well considering the extremes, towards the centre of the graph certain fibre species such as miscanthus, abaca, and sisal deviate considerably from the best fit curve.

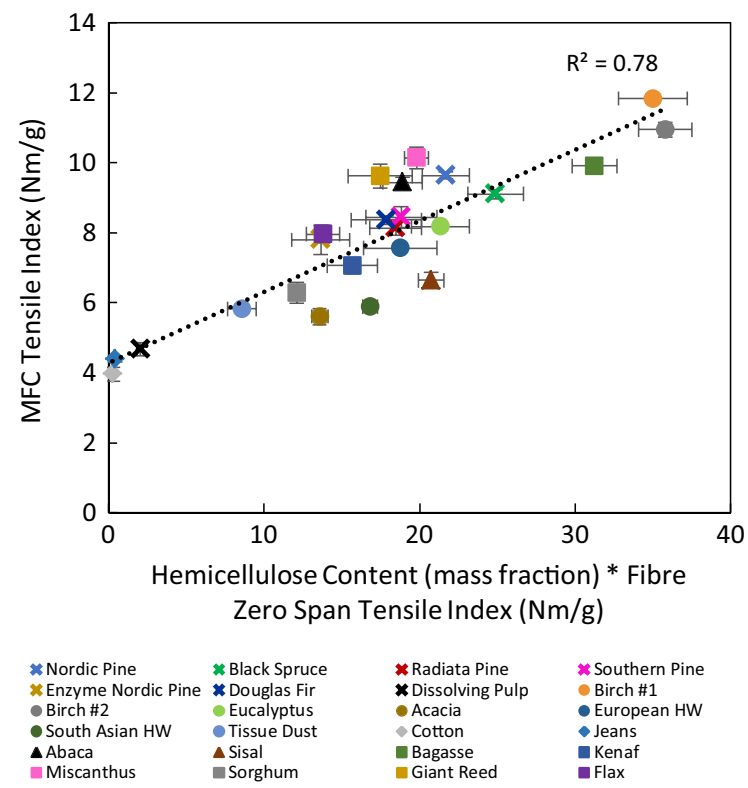

Fig. 5 The correlation between the product of the hemicellulose content and zero-span tensile index of the unground fibres, with the resultant MFC tensile index when ground to the standard energy input
A particularly notable comparison can be made between Nordic pine and enzyme-treated Nordic pine fibres, which are essentially identical in all ways measured except the zero-span tensile index, which has been greatly degraded in the case of the latter. The enzyme-treated variant consequently has much lower MFC particle length and lower MFC tensile strength. This is evidence that the correlation seen with zerospan tensile index is causal.

Despite the increase in data spread compared to when MFC length is used directly, Fig. 5 shows a good fit for most fibre species, and this relationship comes with the important advantage that both predictive parameters are fibre properties that can be measured relatively easily without having to produce the MFC first. This relationship would be a practical tool for shortlisting a large number of fibre species, in order to determine which are worth pursuing to use as a feed for MFC production.

\section{Conclusions}

In this work, microfibrillated cellulose was produced from twenty-four fibre species using a stirred media detritor. The product was used in a composite with calcium carbonate mineral to form nanopaper sheets, which were tested to assess the tensile index. The hemicellulose content of the fibres prior to MFC production was measured and was found to correlate moderately with the tensile index of the respective MFC produced. Additionally, SEM images show that higher hemicellulose fibres result in the liberation of microfibrils with finer widths, which is consistent with what others have reported using alternative MFC production methods.

Various geometric parameters of the fibres such as length and width were measured with a fibre analyser and did not correlate with the MFC tensile index. However, measurements of the apparent length of the MFC product particles were taken with this equipment, and multiplying this length by the hemicellulose content resulted in a parameter that correlated strongly with MFC tensile index. The Page Equation was applied and modified to give this correlation some theoretical basis, with some of the parameters in the bonding term being substituted with hemicellulose content and MFC length. It was found that the addition of a constant $\sigma_{0}$ to represent the residual strength in the 
absence of hemicellulose was required for the model to fit the data.

In order to make this model useful, the zero-span tensile strength of the initial fibres was used as a proxy for the MFC length, since it appears that the frequency of flaws in the fibril structures that is represented by the zero-span tensile strength affects the particle lengths when MFC is produced. This replacement resulted in a weaker fit, but still a substantial improvement over hemicellulose content alone. From this work, it is shown that measurements of the hemicellulose content and zero-span tensile strength of pulp fibres can be used for a reasonably accurate prediction of the resultant MFC tensile strength, providing a facile method to aid in the selection of cellulose sources for use as a feedstock for microfibrillated cellulose production.

\section{Compliance with ethical standards}

Conflict of interest The authors declare that they have no conflict of interest.

Open Access This article is licensed under a Creative Commons Attribution 4.0 International License, which permits use, sharing, adaptation, distribution and reproduction in any medium or format, as long as you give appropriate credit to the original author(s) and the source, provide a link to the Creative Commons licence, and indicate if changes were made. The images or other third party material in this article are included in the article's Creative Commons licence, unless indicated otherwise in a credit line to the material. If material is not included in the article's Creative Commons licence and your intended use is not permitted by statutory regulation or exceeds the permitted use, you will need to obtain permission directly from the copyright holder. To view a copy of this licence, visit http://creativecommons.org/licenses/by/4.0/.

\section{References}

Alila S, Besbes I, Vilar MR, Mutjé P, Boufi S (2013) Nonwoody plants as raw materials for production of microfibrillated cellulose (MFC): a comparative study. Ind Crop Prod 41:250-259

Arola S, Malha JM, Laaksonen P, Lille M, Linder MB (2013) The role of hemicellulose in nanofibrillated cellulose networks. Soft Matter 9:1319-1326

Bolam FM (1965) Stuff preparation for paper and paperboard making: monographs on paperboard and papermaking. Pergamon

Chaker A, Alila S, Mutjé P, Vilar MR, Boufi S (2013) Key role of the hemicellulose content and the cell morphology on the nanofibrillation effectiveness of cellulose pulps. Cellulose 20(6):2863-2875
Chinga-Carrasco G (2011) Cellulose fibres, nanofibrils, and microfibrils: the morphological sequence of MFC components from a plant physiology and fibre technology point of view. Nanoscale Res Lett 6:417

Courchene CE, Peter GF, Litvay J (2006) Cellulose microfibril angle as a determinant of paper strength and hygroexpansivity in Pinus Taeda L. Wood Fiber Sci 38(1):112-120

Desmaisons J, Boutonnet E, Rueff M, Dufresne A, Bras J (2017) A new quality index for benchmarking of different cellulose nanofibrils. Carbohydr Polym 174:318-329

Duchesne I, Hult E, Molin U, Daniel G, Iversen T, Lennholm H (2001) The influence of hemicellulose on fibril aggregation of kraft pulp fibres as revealed by FE-SEM and CP/MAS 13C-NMR. Cellulose 8(2):103-111

Ebringerová A (2005) Structural Diversity and Application Potential of Hemicelluloses. Macromol Symp 232(1):1-12

El-Hosseiny F, Page DH (1975) The mechanical properties of single wood pulp fibres: Theories of strength. J Fiber Sci Technol 8(1):21-31

Fengel D, Wegener G (1983) Wood-chemistry, ultrastructure, reactions. De Gruyter

Herrick FW, Casebier RL, Hamilton JK, Sandberg KR (1983) Microfibrillated cellulose: morphology and accessibility. J Appl Polym Sci: Appl Polym Symp 37:797-813

Horn RA (1974) Morphology of pulp fiber from softwoods and influence on paper strength. Research Paper FPL 242, Forest Product Laboratory, Forest Service, United States Department of Agriculture

Horn RA (1978) Morphology of pulp fiber from hardwoods and influence on paper strength. Research Paper FPL 312, Forest Product Laboratory, Forest Service, United States Department of Agriculture

Hult E-L, Larsson PT, Iversen T (2001) Cellulose fibril aggregation-an inherent property of kraft pulps. Polymer 42(8):3309-3314

Husband JC, Svending P, Skuse DR, Motsi T, Likitalo M, Coles A, Fiberlean Technologies Ltd. (2015) Paper filler composition. U.S. Pat. US9127405B2

Iwamoto S, Abe K, Yano H (2008) The Effect of Hemicelluloses on Wood Pulp Nanofibrillation and Nanofiber Network Characteristics. Biomacromol 9(3):1022-1026

Jonoobi M, Oladi R, Davoudpour Y, Oksman K, Dufresne A, Hamzeh Y, Davoodi R (2015) Different preparation methods and properties of nanostructures cellulose from various natural resources and residues: a review. Cellulose 22(2):935-969

Joutsimo O, Wathén R, Tamminen T (2005) Effects of fiber deformations on pulp sheet properties and fiber strength. Paperi Ja Puu/Paper and Timber 87(6)

Klemm D, Heublein B, Fink HP, Bohn A (2005) Cellulose: Fascinating Biopolymer and Sustainable Raw Material. Angew Chem Int Ed 44(22):3358-3393

Klemm D, Kramer F, Moritz S, Lindström T, Ankerfors M, Gray D, Dorris A (2011) Nanocelluloses: a new family of naturebased materials. Angew Chem Int Ed 50(24):5438-5466

Lahtinen P, Liukkonen S, Pere J, Sneck A, Kangas H (2014) A Comparative Study of Fibrillated Fibers from Different Mechanical and Chemical Pulps. BioResources 9(2):2115-2127

Liitiä T, Maunu SL, Hortling B, Tamminen T, Pekkala O, Varhimo A (2003) Cellulose crystallinity and ordering of 
hemicelluloses in pine and birch pulps as revealed by solidstate NMR spectroscopic methods. Cellulose 10(4): 307-316

Lindström T, Fellers C, Ankerfors M, Nordmark GG (2016) On the nature of joint strength of paper-effect of dry strength agents-Revisiting the Page equation. Nord Pulp Pap Res J 31(3):459-468

Mäkelä P, Bergnor E, Wallbäcks L, Öhman F (2010) Sorption of birch xylan to softwood kraft pulps and its influence on the tensile properties of previously-dried papers under different papermaking conditions. Innventia Report No. 121 2nd Version

Meyer V, Lecourt M, Tapin-Lingua S, Petit-Conil M (2018) Production of Microfibrillated Cellulose (MFC) from Chemical and Mechanical Pulps to Enhance TMP and DIP Paper Properties. Cellulose 72(3):6-16

Nevell TP, Nugawela D (1987) Effect of treatment with very dilute acids on the wet tensile strength and chemical properties of paper. Carbohydr Polym 7(3):169-181

Page DH (1969) A theory for the tensile strength of paper. Tappi J 52(4):674-681

Petroudy SRD, Ghasemian A, Resalati H, Syverud K, ChingaCarrasco G (2015) The effect of xylan on the fibrillation efficiency of DED bleached soda bagasse pulp and on nanopaper characteristics. Cellulose 22(1):385-395

Phipps J, Larson T, Ingle D, Eaton H (2017) The Effect of Microfibrillated Cellulose on the Strength and Light Scattering of Highly Filled Papers. In: Batchelor W, Söderberg D (eds) Advances in Pulp and Paper Research: Transactions of the 16th Fundamental Research Symposium 231-254

Prado KS, Spinacé MAS (2015) Characterization of Fibers from Pineapple's Crown, Rice Husks and Cotton Textile Residues. Mat Res 18(3):530-537

Scandinavian Pulp, Paper and Board Testing Committee (2009) Carbohydrate composition. Test Method SCAN CM 71:09

Shatkin JA, Wegner TH, Bilek EM, Cowie J (2014) Market projections of cellulose nanomaterial-enabled productspart 1: applications. TAPPI J 13(5):9-16

Siró I, Plackett D (2010) Microfibrillated cellulose and new nanocomposite materials: a review. Cellulose 17(3): 459-494
Spence KL, Venditti RA, Habibi Y, Rojas OJ, Pawlak JJ (2010) The effect of chemical composition on microfibrillar cellulose films from wood pulps: Mechanical processing and physical properties. Bioresour Technol 101(15): 5961-5968

Technical Association of the Pulp and Paper Industry (1988) Acid-Insoluble Lignin in Wood and Pulp. Test Method T 222 om- 88

Technical Association of the Pulp and Paper Industry (2007) Zero-span breaking strength of pulp (dry zero-span tensile). Test Method T $231 \mathrm{~cm}-07$

Technical Association of the Pulp and Paper Industry (2012) Forming Handsheets for Physical Tests of Pulp. Test Method T $205 \mathrm{~cm}-12$

Turbak AF, Snyder FW, Sandberg KR (1983) Microfibrillated cellulose, a new cellulose product: properties, uses, and commercial potential. J Appl Polym Sci: Appl Polym Symp 37:815-827

Uetani K, Yano H (2012) Zeta potential time dependence reveals the swelling dynamics of wood cellulose nanofibrils. Langmuir 28(1):818-827

Varanasi S, Chiam HH, Batchelor W (2012) Application and interpretation of zero and short-span testing on nanofibre sheet materials. Nord Pulp Pap Res J 27(2):343-506

Virtanen T, Maunu SL, Tamminen T, Hortling B, Liitiä T (2008) Changes in fiber ultrastructure during various kraft pulping conditions evaluated by 13C CPMAS NMR spectroscopy. Carbohydr Polym 73(1):156-163

Wathén R (2006) Studies on fiber strength and its effect on paper properties. PhD Thesis, King's College London. ISSN $1457-6252$

Zeng X, Retulainen E, Heinemann S, Fu S (2012) Fibre deformations induced by different mechanical treatments and their effect on zero-span strength. Nord Pulp Pap Res J 27(2):335-342

Publisher's Note Springer Nature remains neutral with regard to jurisdictional claims in published maps and institutional affiliations. 